\title{
First-principles study of crystal structure, electronic structure, and second- harmonic generation in a polar double perovskite $\mathrm{Bi}_{2} \mathrm{ZnTiO}_{6}$
}

\author{
Sheng Ju ${ }^{1,2, a)}$ and Guang-Yu Guo ${ }^{1, b)}$ \\ ${ }^{1}$ Department of Physics and Center of Theoretical Sciences, National Taiwan University, Taipei 106, Taiwan \\ ${ }^{2}$ Department of Physics, Suzhou University, Suzhou 215006, China
}

(Received 18 April 2008; accepted 2 October 2008; published online 18 November 2008)

\begin{abstract}
Within the density functional theory with the generalized gradient approximation, we present a systematic $a b$ initio investigation of crystal structure, electronic structure, and linear and nonlinear optical responses in a polar double perovskite $\mathrm{Bi}_{2} \mathrm{ZnTiO}_{6}$. The effect of $B$-site ordering is explored by comparing three possible configurations: $A$-type with alternative $\mathrm{Zn}$ and Ti layers stacking along the $c$ axis; $C$-type with $\mathrm{Zn}$ and $\mathrm{Ti} c$ axis chains; and $G$-type with every $\mathrm{Zn}(\mathrm{Ti})$ atoms is surrounded by its nearby six $\operatorname{Ti}(\mathrm{Zn})$ atoms. It is found that the system with $G$-type $B$-site ordering is energetically favorable, which is lower in the total energies of 0.055 and $0.133 \mathrm{eV} /$ formula unit than $C$-type and $A$-type, respectively. Optical calculations indicate that all the three configurations show large second-harmonic generation (SHG) coefficients, and the largest static SHG observed in the $C$-type system reaches $123\left(10^{-9} \mathrm{esu}\right)$, the value of which is much larger than ever known polar oxides, e.g., $72\left(10^{-9}\right.$ esu $)$ in $\mathrm{LiNbO}_{3}$. The predicted significant nonlinear optical properties are consistent with the calculated high tetragonality as well as the large off-center displacement of $\mathrm{Zn}$, $\mathrm{Ti}$, and $\mathrm{Bi}$ atoms. In particular, a large off-center displacement greater than $0.5 \AA$ in $\mathrm{Zn}$ atoms is revealed for the first time. A further microscopic picture is presented via the successful connection of the prominent feature of $\mathrm{SHG}$ in $\mathrm{Bi}_{2} \mathrm{ZnTiO}_{6}$ with that of the linear optical dielectric function in terms of single-photon and double-photon resonances. Our calculations demonstrate the promising application of $\mathrm{Bi}_{2} \mathrm{ZnTiO}_{6}$ in optoelectronics. (C) 2008 American Institute of Physics.
\end{abstract}

[DOI: 10.1063/1.3009623]

\section{INTRODUCTION}

Materials with double perovskite structure have displayed many interesting fundamental physics and technological applications, including colossal low-field magnetoresistance in $\mathrm{Sr}_{2} \mathrm{FeMoO}_{6},{ }^{1}$ giant magnetocapacitance in $\mathrm{La}_{2} \mathrm{NiMnO}_{6},{ }^{2}$ and antiferromagnetic half metals. ${ }^{3}$ Recently, multiferroic $\mathrm{Bi}_{2} \mathrm{FeCrO}_{6}$ (Refs. 4-6 and $\mathrm{Bi}_{2} \mathrm{NiMnO}_{6}$ (Refs. 7 and 8) with highly distorted double perovskite structure have drawn great interest due to the coexistence of robust electric polarization $P$ and magnetic moment $M$ in a single phase. Besides the above mentioned multiferroics, which are composed of magnetic ions, $\mathrm{Fe}, \mathrm{Cr}, \mathrm{Mn}, \mathrm{Ni}$, and $\mathrm{Co},{ }^{9}$ (double) perovskite structures of $\mathrm{BiBO}_{3}$ or $\mathrm{Bi}_{2} \mathrm{BB}^{\prime} \mathrm{O}_{6}$ with nonmagnetic $B$ and $B^{\prime}$ ions (Al, $\mathrm{In}, \mathrm{Sc}, \mathrm{Ga}, \mathrm{Zn} / \mathrm{Ti}$, and $\mathrm{Mg} / \mathrm{Ti}$ ) also display interesting physics, including piezoelectricity, ferroelectricity, and nonlinear optical coefficients. ${ }^{10-20}$ They are important as the replacement of lead-free or low lead materials, possessing similar "inert-pair" $6 s^{2}$ electronic configuration with $\mathrm{Pb}^{2+}$, but quite environmentally benign in their oxide form. In particular, a recently synthesized double perovskite $\mathrm{Bi}_{2} \mathrm{ZnTiO}_{6}$ is found to have a large $c / a$ ratio of 1.2, where $a$ and $c$ are the lattice constants of a simple $A B O_{3}$ perovskite. ${ }^{19}$ The calculated ionic polarization in a point charge model reaches as high as $103 \mu \mathrm{C} \mathrm{cm}^{-2}$, much larger than ever known polar oxides, e.g., $32 \mu \mathrm{C} \mathrm{cm}^{-2}$ in $\mathrm{BaTiO}_{3}$,

\footnotetext{
a)Electronic mail: jusheng@suda.edu.cn.

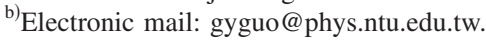

$50 \mu \mathrm{C} \mathrm{cm}^{-2}$ in $\mathrm{PbTiO}_{3}, \quad 70 \mu \mathrm{C} \mathrm{cm}^{-2}$ in $\mathrm{LiNbO}_{3}$, or $90 \mu \mathrm{C} \mathrm{cm}^{-2}$ in multiferroic $\mathrm{BiFeO}_{3}$. On the other hand, experiment on the solid solutions of $\left[\mathrm{Bi}_{2} \mathrm{ZnTiO}_{6}\right]_{x}\left[\mathrm{PbTiO}_{3}\right]_{1-x}$ with $1-x \leqslant 0.6$ also exhibits a significant increase in the tetragonal distortion and $T_{C}$. In particular, the tetragonal $\mathrm{c} / \mathrm{a}$ ratio of 1.11 and Curie temperature $T_{C}$ in excess of $700{ }^{\circ} \mathrm{C}$ are found when $x=0.40 .^{20}$

It is noted that polar oxides $\mathrm{BiAlO}_{3}$ and $\mathrm{BiInO}_{3}$ exhibit large second-harmonic generation (SHG) signals, about 150170 times larger than that in quartz. ${ }^{13,14}$ Therefore, it is natural to study the nonlinear optical response in $\mathrm{Bi}_{2} \mathrm{ZnTiO}_{6}$, which might be significant for its unusual polar structure. In fact, for ferroelectrics, the free energy can be written as

$$
F=F_{0}+\frac{1}{2} a P^{2}+\frac{1}{4} b P^{4}-P E,
$$

where $P=P_{0}+p$. With a given electric field $E$, it is assumed that $P$ is one dimensional and along electric field direction. Following the conventional form of nonlinear optics, we express the electric field response $p$ as a power series in $E$, $\chi^{(1)} E+\chi^{(2)} E^{2}+\chi^{(3)} E^{3}+\ldots$, with disregard of tensor suffices for now. Here, $\chi^{(2)}$ appears only in the system lacking inversion symmetry. According to the condition of steady state $\partial F / \partial P=0, P$ is determined by

$$
b P^{3}+a P-E=0 .
$$

If the contribution of nonlinear part to the electric polarization is small, 


$$
\chi^{(1)}=\frac{1}{a+3 b P_{0}^{2}}
$$

and

$$
\chi^{(2)}=\frac{-3 b P_{0}}{\left(a+3 b P_{0}^{2}\right)^{3}}=\frac{3 b P_{0}}{8 a^{3}} \propto P_{0} .
$$

Therefore, the larger spontaneous electric polarization $P_{0}$, the stronger nonlinear susceptibilities $\chi^{(2)}$. The significant electric polarization of $103 \mu \mathrm{C} \mathrm{cm}^{-2}$ found in $\mathrm{Bi}_{2} \mathrm{ZnTiO}_{6}$ (Ref. 19) indicates that the nonlinear optical coefficients should be significant.

The density functional theory with modern theory of electric polarization ${ }^{21,22}$ has been successfully applied in predicting crystal structures and electric polarizations in polar $\mathrm{Bi}_{2} \mathrm{FeCrO}_{6},{ }^{23} \mathrm{Bi}_{2} \mathrm{NiMnO}_{6}{ }^{24} \mathrm{BiFeO}_{3}{ }^{25} \mathrm{BiAlO}_{3}{ }^{26}$ and alloys of $\left(\mathrm{BiScO}_{3}\right)_{1-x}-\left(\mathrm{PbTiO}_{3}\right)_{x}$ as well as $\left[\mathrm{Bi}_{2} \mathrm{ZnTiO}_{6}\right]_{x}\left[\mathrm{PbTiO}_{3}\right]_{1-x}$ (with $x=0.25$ and 0.5 ), ${ }^{27}$ but the $a b$ initio study of crystal structure and electronic structure of pure $\mathrm{Bi}_{2} \mathrm{ZnTiO}_{6}$ is still absent, not mentioning its nonlinear optical response. In this paper, within the density functional theory with the generalized gradient approximation (GGA), we have performed a systematic $a b$ initio investigation of crystal structure, electronic structure, and nonlinear optical properties of $\mathrm{Bi}_{2} \mathrm{ZnTiO}_{6}$. In particular, since current experimental technique could not determine the long-range or short-range orderings of $\mathrm{Zn}$ and Ti cations over $B$-site, which is the important information for the discussion on the structure in terms of the $B$-site environment, we explore the effect of $B$-site ordering on the crystal structure and corresponding electronic and optical properties. As shown in Figs. 1(a)-1(c), we consider three types of $B$-site ordering: alternative $\mathrm{Zn}$ and $\mathrm{Ti}$ layers along $c$ the axis ( $A$-type ordering); $\mathrm{Zn}$ and $\mathrm{Ti}$ atoms chains along the $c$ axis ( $C$-type ordering); and every $\mathrm{Zn}(\mathrm{Ti})$ atoms is surrounded by its nearby six $\mathrm{Ti}(\mathrm{Zn})$ atoms ( $G$-type ordering). In the practical calculations, $\sqrt{2} a \times \sqrt{2} a \times 2 c$ supercells are adopted to account for all three possible $B$-site ordering structures. The detailed fullrelaxed lattice parameters and ionic positions are displayed in Table I. Our calculations presented below will give strong evidence on the correlation between the highly distorted structures and significantly large SHG coefficients in $\mathrm{Bi}_{2} \mathrm{ZnTiO}_{6}$. Furthermore, by connecting the nonlinear optical response with linear part, we also present a microscopic picture of SHG in $\mathrm{Bi}_{2} \mathrm{ZnTiO}_{6}$. Our calculations suggest the promising application of $\mathrm{Bi}_{2} \mathrm{ZnTiO}_{6}$ in future optoelectronics.

\section{COMPUTATIONAL METHOD}

Our $a b$ initio calculations for $\mathrm{Bi}_{2} \mathrm{ZnTiO}_{6}$ are performed using the highly accurate full-potential projector augmented wave (PAW) method, ${ }^{28}$ as implemented in the Vienna $a b$ initio Simulation Package (VASP) package. ${ }^{29}$ They are based on density functional theory with GGA. A large plane-wave cutoff of $500 \mathrm{eV}$ is used throughout. PAW potentials are used to describe the electron-ion interaction, with 15 valence electrons for $\mathrm{Bi}\left(5 d^{10} 6 s^{2} 6 p^{3}\right), 12$ for $\mathrm{Zn}\left(3 d^{10} 4 s^{2}\right), 10$ for $\mathrm{Ti}$ $\left(3 p^{6} 3 d^{2} 4 s^{2}\right)$, and 6 for $\mathrm{O}\left(2 s^{2} 2 p^{4}\right)$. Brillouin zone integra-
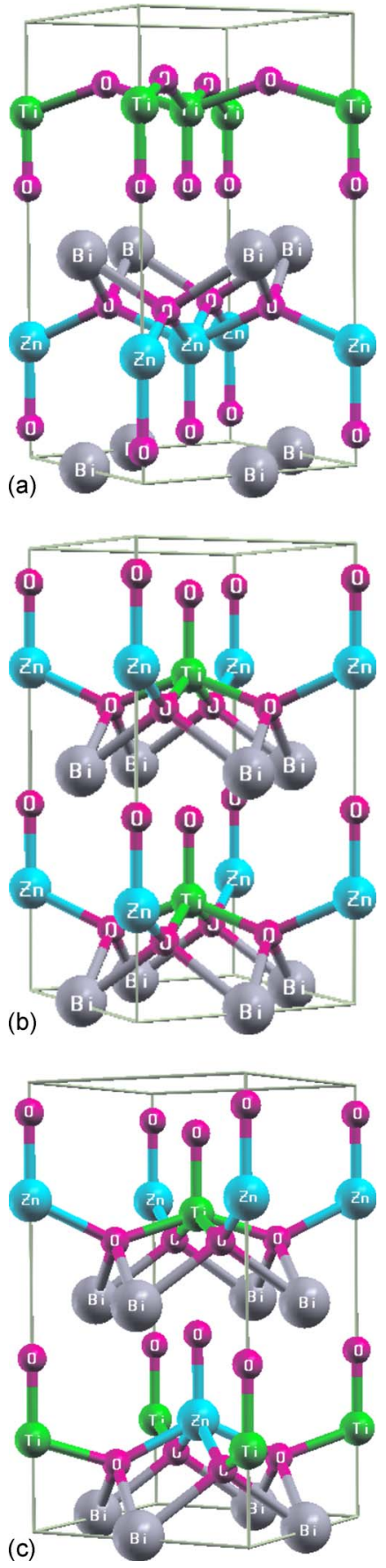

FIG. 1. (Color online) Crystal structure of $\mathrm{Bi}_{2} \mathrm{ZnTiO}_{6}$. (a) A-type. (b) $C$-type. (c) $G$-type.

tions are performed with tetrahedron method in a $8 \times 8 \times 4$ Monkhorst-Pack $k$-point mesh centered at $\Gamma$. The convergence criterion for the electronic energy is $10^{-6} \mathrm{eV}$. The atomic positions and lattice constants were fully relaxed by a conjugate gradient technique. The final theoretical structures were obtained when the maximum atomic force and maximum stress is less than $0.004 \mathrm{eV} / \AA$ and $0.04 \mathrm{kbar}$, respectively. In the self-consistent band structure and following optical properties calculations, a much denser $k$-points grid $16 \times 16 \times 8$ is adopted with $225 k$-points in the irreducible Brillouin zone. Also 20 bands per atom are adopted in the optical calculations. The density of states (DOS) is evaluated 
TABLE I. Structure parameters of the experimental (Ref. 19), and calculated $A$-type, $C$-type, and $G$-type $\mathrm{Bi}_{2} \mathrm{ZnTiO}_{6}$. The space group is $P 4 \mathrm{~mm}$.

\begin{tabular}{|c|c|c|c|c|c|}
\hline & & $\begin{array}{c}\text { Expt. } \\
(a \times a \times c)\end{array}$ & $\begin{array}{c}A \text {-type } \\
(a \times a \times 2 c)\end{array}$ & $\begin{array}{c}C \text {-type } \\
(\sqrt{2} a \times \sqrt{2} a \times c)\end{array}$ & $\begin{array}{c}G \text {-type } \\
(\sqrt{2} a \times \sqrt{2} a \times 2 c)\end{array}$ \\
\hline \multicolumn{6}{|c|}{ (i) Lattice constants } \\
\hline$\sqrt{2} a$ & $(\mathrm{~nm})$ & 0.5405 & 0.5353 & 0.5306 & 0.5327 \\
\hline $2 c$ & $(\mathrm{~nm})$ & 0.9256 & 0.9955 & 0.9985 & 0.9822 \\
\hline $\mathrm{Bi}$ & $\begin{array}{l}1 a \\
1 a\end{array}$ & $(0,0,0)$ & $\begin{array}{c}\text { (ii) Atomic po } \\
(0,0,0) \\
(0,0,0.4677)\end{array}$ & $(0,0.5,0)$ & $(0,0.5,0)$ \\
\hline $\mathrm{Zn}$ & $1 b$ & $(0.5,0.5,0.5662)$ & $(0.5,0.5,0.261)$ & $(0,0,0.553)$ & $(0,0,0.269)$ \\
\hline $\mathrm{Ti}$ & $1 b$ & $(0.5,0.5,0.5662)$ & $(0.5,0.5,0.788)$ & $(0.5,0.5,0.586)$ & $(0.5,0.5,0.302)$ \\
\hline \multirow[t]{3}{*}{$\mathrm{O}_{\|}$} & $2 c$ & $(0.5,0,0.692)$ & $(0,0.5,0.348)$ & & \\
\hline & $2 c$ & & $(0,0.5,0.849)$ & & \\
\hline & $2 b$ & & & $(0.257,0.257,0.727)$ & $(0.251,0.251,0.362)$ \\
\hline \multirow[t]{2}{*}{$\mathrm{O}_{\perp}$} & $2 c$ & $(0.5,0.5,0.184)$ & $(0.5,0.5,0.064)$ & $(0,0,0.171)$ & $(0,0,0.075)$ \\
\hline & $2 c$ & & $(0.5,0.5,0.616)$ & $(0.5,0.5,0.229)$ & $(0.5,0.5,0.120)$ \\
\hline
\end{tabular}

from the self-consistent band structure by tetrahedral method. ${ }^{30}$

The optical properties are calculated based on the independent-particle approximation, i.e., the excitonic effects and the local-field corrections are neglected. ${ }^{31-33}$ The imaginary part of the dielectric function due to direction interband transitions is given by the Fermi golden rule, i.e., ${ }^{31}$

$$
\varepsilon_{a a}^{\prime \prime}=\frac{4 \pi^{2}}{\Omega \omega^{2}} \sum_{i \in V B, j \in C B} \sum_{\mathbf{k}} w_{\mathbf{k}}\left|p_{i j}^{a}\right|^{2} \delta\left(\varepsilon_{\mathbf{k}_{j}}-\varepsilon_{\mathbf{k}_{i}}-\omega\right),
$$

where $\Omega$ is the unit-cell volume and $\omega$ is the photon energy. $\mathrm{VB}$ and $\mathrm{CB}$ denote the conduction and valence bands, respectively. The dipolar transition matrix elements $p_{i j}^{a}$ $=\left\langle\mathbf{k}_{j}\left|p_{a}\right| \mathbf{k}_{i}\right\rangle$ are obtained from the self-consistent band structures within the PAW formalism. Here $\left|\mathbf{k}_{n}\right\rangle$ is the $n$th Bloch state wave function with crystal momentum $\mathbf{k}$ and $a$ denotes the Cartesian component. The real part of the dielectric function is obtained from $\varepsilon^{\prime \prime}$ by a Kramers-Kronig transformation $^{31}$

$$
\varepsilon^{\prime}(\omega)=1+\frac{2}{\pi} \mathbf{P} \int_{0}^{\infty} d \omega \frac{\omega^{\prime} \varepsilon^{\prime \prime}\left(\omega^{\prime}\right)}{\omega^{\prime 2}-\omega^{2}} .
$$

Here $\mathbf{P}$ is the principle value of the integral.

Given the complex dielectric function $\varepsilon(\omega)=\varepsilon^{\prime}(\omega)$ $+i \varepsilon^{\prime \prime}(\omega)$, all the other linear optical properties can be calculated, e.g., linear refractive index

$$
n(\omega)=\left(\frac{\sqrt{\varepsilon^{\prime 2}(\omega)+\varepsilon^{\prime \prime 2}(\omega)}+\varepsilon^{\prime}(\omega)}{2}\right)^{1 / 2}
$$

and the linear absorption coefficient is related to $\varepsilon^{\prime \prime}$ by $\alpha=\varepsilon^{\prime \prime} \omega /(n c)$, where $c$ is the velocity of light in the vacuum. The electron energy-loss function is given by $\operatorname{Im}(-1 / \varepsilon)$.

The imaginary part of the second-order optical susceptibility due to the direct interband transitions is given by ${ }^{33}$

$$
\begin{aligned}
\chi_{a b c}^{\prime \prime(2)}(-2 \omega, \omega, \omega)= & \chi_{a b c, \mathrm{VE}}^{\prime \prime(2)}(-2 \omega, \omega, \omega) \\
& +\chi_{a b c, \mathrm{VH}}^{\prime \prime(2)}(-2 \omega, \omega, \omega),
\end{aligned}
$$

where the contribution due to the so-called virtual-electron (VE) process is ${ }^{33}$

$$
\begin{aligned}
\chi_{a b c, \mathrm{VE}}^{\prime \prime(2)}(-2 \omega, \omega, \omega) & =\frac{\pi}{2 \Omega} \sum_{i \in V B} \sum_{j l \in C B} \sum_{\mathbf{k}} w_{\mathbf{k}}\left\{\frac{\operatorname{Im}\left[p_{j l}^{a}\left\langle p_{l i}^{b} p_{i j}^{c}\right\rangle\right]}{\varepsilon_{l i}^{3}\left(\varepsilon_{l i}+\varepsilon_{j i}\right)} \delta\left(\varepsilon_{l i}-\varepsilon\right)\right. \\
& -\frac{\operatorname{Im}\left[p_{i j}^{a}\left\langle p_{j l}^{b} p_{l i}^{c}\right\rangle\right]}{\varepsilon_{l i}^{3}\left(2 \varepsilon_{l i}-\varepsilon_{j i}\right)} \delta\left(\varepsilon_{l i}-\omega\right) \\
& \left.+\frac{16 \operatorname{Im}\left[p_{i j}^{b}\left\langle p_{j l}^{b} p_{l i}^{c}\right\rangle\right]}{\varepsilon_{j i}^{3}\left(2 \varepsilon_{l i}^{3}-\varepsilon_{j i}^{3}\right)} \delta\left(\varepsilon_{j i}-2 \omega\right)\right\},
\end{aligned}
$$

and that due to the virtual-hole $(\mathrm{VH})$ process is ${ }^{33}$

$$
\begin{aligned}
\chi_{a b c, \mathrm{VH}}^{\prime \prime(2)}(-2 \omega, \omega, \omega) & \\
= & -\frac{\pi}{2 \Omega} \sum_{i \in V B} \sum_{j l \in C B} \sum_{\mathbf{k}} w_{\mathbf{k}}\left\{\frac{\operatorname{Im}\left[p_{j l}^{a}\left\langle p_{l i}^{b} p_{i j}^{c}\right\rangle\right]}{\varepsilon_{l i}^{3}\left(\varepsilon_{l i}+\varepsilon_{j i}\right)} \delta\left(\varepsilon_{l i}-\omega\right)\right. \\
& -\frac{\operatorname{Im}\left[p_{i j}^{a}\left\langle p_{j l}^{b} p_{l i}^{c}\right\rangle\right]}{\varepsilon_{l i}^{3}\left(2 \varepsilon_{l i}-\varepsilon_{j i}\right)} \delta\left(\varepsilon_{l i}-\omega\right) \\
& \left.+\frac{16 \operatorname{Im}\left[p_{i j}^{b}\left\langle p_{j l}^{b} p_{l i}^{c}\right\rangle\right]}{\varepsilon_{j i}^{3}\left(2 \varepsilon_{l i}^{3}-\varepsilon_{j i}^{3}\right)} \delta\left(\varepsilon_{j i}-2 \omega\right)\right\} .
\end{aligned}
$$

Here $\varepsilon_{j i}=\varepsilon_{\mathbf{k} i}-\varepsilon_{\mathbf{k} i}$ and $\left\langle p_{j l}^{b} p_{l i}^{c}\right\rangle=\frac{1}{2}\left(p_{j l}^{b} p_{l i}^{c}+p_{j l}^{b} p_{l i}^{c}\right)$. The real part of the second-order optical susceptibility is then obtained from the imaginary part by a Kramers-Kronig transformation $^{33}$

$$
\chi^{\prime(2)}(-2 \omega, \omega, \omega)=\frac{2}{\pi} \mathbf{P} \int_{0}^{\infty} d \omega^{\prime} \frac{\omega^{\prime} \chi^{\prime(2)}\left(2 \omega^{\prime}, \omega^{\prime}, \omega^{\prime}\right)}{\omega^{\prime 2}-\omega^{2}} .
$$

The electronic part of linear electro-optical coefficient is $r_{a b c}^{e}(\omega)$, which is connected to the second-order optical susceptibility $\chi_{a b c}^{(2)}(-2 \omega, \omega, \omega)$ through the relation ${ }^{33}$ 

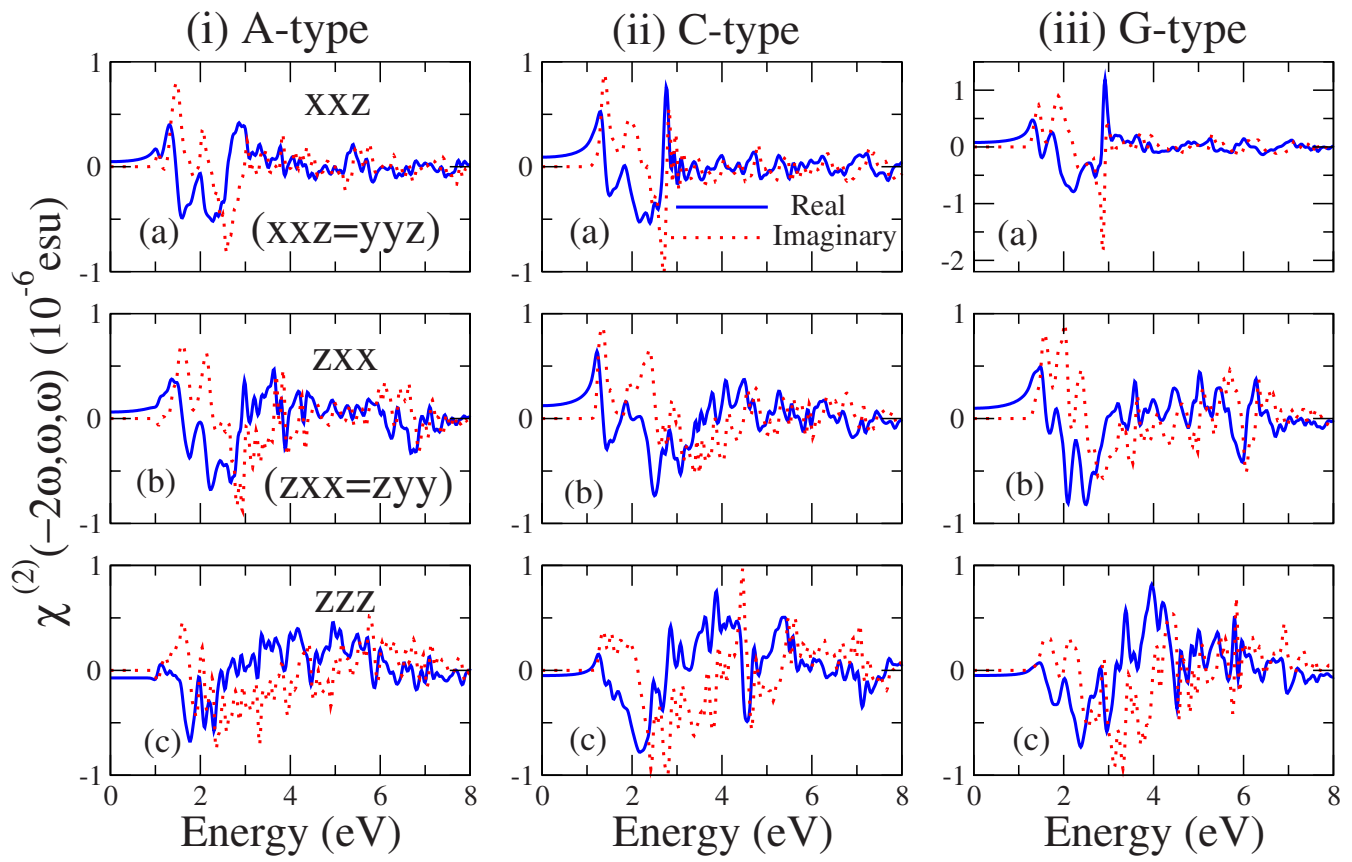

FIG. 2. (Color online) SHG susceptibilities. From top to bottom are the components $\chi_{x x z}^{(2)}\left(\chi_{y y z}^{(2)}\right), \chi_{z z z}^{(2)}\left(\chi_{z y y}^{(2)}\right)$, and $\chi_{z z z}^{(2)}$ respectively. From left to right are $A$-type, $C$-type, and $G$-type, respectively. Blue solid line is for real part and red dotted line is for imaginary part.

$$
\chi_{a b c}^{(2)}(-\omega, \omega, 0)=-\frac{1}{2} n_{a}^{2}(\omega) n_{b}^{2}(\omega) r_{a b c}^{e}(\omega),
$$

where $n(\omega)=\sqrt{\varepsilon(\omega)}$ is the refraction index in the $a$ direction. In the low frequency limit,

$$
\lim _{\omega \rightarrow 0} \chi_{a b c}^{(2)}(-2 \omega, \omega, \omega)=\lim _{\omega \rightarrow 0} \chi_{a b c}^{(2)}(-\omega, \omega, 0) .
$$

Therefore,

$$
r_{a b c}^{e}(0)=-\frac{2}{n_{a}^{2}(0) n_{b}^{2}(0)} \lim _{\omega \rightarrow 0} \chi_{a b c}^{(2)}(-2 \omega, \omega, \omega)
$$

It is noted that for the photon energy $\hbar \omega$ well below the band gap, the linear electro-optical coefficient $r_{a b c}^{e}(\omega) \approx r_{a b c}^{e}(0)$, which is due to the fact that both the $\chi^{(2)}(-2 \omega, \omega, \omega)$ and $n(\omega)$ are nearly constant in this low frequency region. In the present calculations, the $\delta$ functions in Eqs. (5), (8), and (9) are approximated by a Gaussian function with $\Gamma=0.1 \mathrm{eV}$. The maximum energy in Eqs. (6) and (10) is set as $50 \mathrm{eV}$ to ensure completeness of the real part of $\varepsilon$ and $\chi^{(2)}$ in the integrals.

\section{RESULTS AND DISCUSSION}

First, let us consider the SHG directly. It is noted that the $P 4 \mathrm{~mm}$ symmetry of $\mathrm{Bi}_{2} \mathrm{ZnTiO}_{6}$ allows five nonvanishing components, i.e., $\chi_{x x z y}^{(2)}, \chi_{y y z}^{(2)}, \chi_{z x x}^{(2)}, \chi_{z y y}^{(2)}$, and $\chi_{z z z}^{(2)}$, with $\chi_{x x z}^{(2)}$ $=\chi_{y y z}^{(2)}$ and $\chi_{z x x}^{(2)}=\chi_{z y y}^{(2)}{ }^{3}$ In Fig. 2, SHG susceptibilities are plotted and compared. From top to bottom are the components $\chi_{x x z}^{(2)}, \chi_{z x x}^{(2)}$, and $\chi_{z z z}^{(2)}$, respectively. From left to right, we present the results of $A$-type, $C$-type, and $G$-type, respectively. It is clear that all the systems show significantly large SHG coefficients. Furthermore, for the photon energy smaller than $0.78 \mathrm{eV}$ (A-type), $1.0 \mathrm{eV}$ (C-type), and $1.16 \mathrm{eV}$ ( $G$-type), $\chi^{(2)}$ is purely dispersive, i.e., real and lossless, suggesting that $\mathrm{Bi}_{2} \mathrm{ZnTiO}_{6}$ has potential application in nonlinear optical devices. The real part remains nearly constant at first, then increases steadily as the increase of photon energy, and finally peaks at the absorption edge. At low energy, $C$-type system shows much larger signals, e.g., at $1 \mathrm{eV}, \chi_{z x x}^{(2)}$ has reached $0.75 \times 10^{-6}$ esu. As the photon energy increases, $G$-type system, i.e., energetically favorable case, exhibits much larger SHG signals, e.g., at $3 \mathrm{eV}, \chi_{x x z}^{(2)}$ has surpassed $1 \times 10^{-6} \mathrm{esu}$, and at $4 \mathrm{eV}, \chi_{z z z}^{(2)}$ is near $1 \times 10^{-6} \mathrm{esu}$. When the energy is further increased, all the systems show the decreased SHG coefficients. From the above consideration, we can find that the energetically favorable $G$-type $\mathrm{Bi}_{2} \mathrm{ZnTiO}_{6}$ has a large second-order nonlinear optical response. Interestingly, these large SHG spectra can be comparable with the responses in GaAs and zigzag BN nanotubes. ${ }^{33}$ The imaginary part of SHG is displayed in Figs. 3-5.

The static SHG susceptibilities, dielectric constant, and corresponding linear electro-optic coefficients are listed in Table II. It is noted that all the components of three systems show large magnitudes $\left(\geqslant 50 \times 10^{-9} \mathrm{esu}\right)$. For comparison, it is noted that in $\mathrm{LiNbO}_{3}$, which is the popular nonlinear optic

TABLE II. Static dielectric constant $(\epsilon)$, second-order susceptibility $\left(\chi^{(2)}\right)$ $\left(10^{-8} \mathrm{esu}\right)$, and linear electro-optical coefficient (electronic part $\left.r^{e}\right)$ $\left(10^{-8} \mathrm{esu}\right)$ of three types of $\mathrm{Bi}_{2} \mathrm{ZnTiO}_{6}$.

\begin{tabular}{lrrr}
\hline \hline & $A$-type & $C$-type & $G$-type \\
\hline$\epsilon_{x x}$ & 6.55 & 6.62 & 6.62 \\
$\epsilon_{z z}$ & 5.69 & 5.75 & 5.75 \\
$\chi_{x x z}^{(2)}$ & 4.86 & 9.15 & 7.82 \\
$\chi_{z x x}^{(2)}$ & 6.24 & 12.28 & 9.84 \\
$\chi_{z z z}^{(2)}$ & -7.18 & -4.99 & -4.91 \\
$r_{x x z}^{e}$ & -0.23 & -0.42 & -0.36 \\
$r_{z x x}^{e}$ & -0.33 & -0.65 & -0.52 \\
$r_{z z z}^{e}$ & 0.44 & 0.30 & 0.30 \\
\hline \hline
\end{tabular}


TABLE III. Total energy (eV/f.u.), c/a ratio, bond length $(\AA)$, and off-center displacement $(\AA)$ in three types of $\mathrm{Bi}_{2} \mathrm{ZnTiO}_{6}$.

\begin{tabular}{lccc}
\hline \hline & $A$-type & $C$-type & $G$-type \\
\hline Total energy & 0.133 & 0.055 & 0 \\
$c / a$ & 1.315 & 1.331 & 1.304 \\
Bi-O (1) & $2.2370 / 2.4144$ & 2.3194 & $2.3211 / 2.3211$ \\
Bi-O (2) & $3.0568 / 2.7519$ & $2.7867 / 2.8881$ & $2.9141 / 2.7648$ \\
Bi-O (3) & $4.2449 / 3.9470$ & 4.0864 & $4.9485 / 4.5831$ \\
Bi off-center displacement & $\mathbf{0 . 9 8 2 1 / 1 . 3 0 3 6}$ & $\mathbf{1 . 1 3}$ & $\mathbf{1 . 1 0}$ \\
Zn-O (1) & 2.0778 & 2.1175 & 2.0987 \\
Zn-O (2) & 1.9667 & 1.9070 & 1.9033 \\
Zn-O (3) & 3.5265 & 3.0856 & 3.4488 \\
Zn off-center displacement & $\mathbf{0 . 7 7 9 9}$ & $\mathbf{0 . 5 8 9 3}$ & $\mathbf{0 . 7 7 2 5}$ \\
Ti-O (1) & 1.9895 & 1.9535 & 1.9674 \\
Ti-O (2) & 1.7099 & 1.7836 & 1.7816 \\
Ti-O (3) & 2.7519 & 3.2090 & 2.6887 \\
Ti off-center displacement & $\mathbf{0 . 5 2 1 0}$ & $\mathbf{0 . 7 1 2 7}$ & $\mathbf{0 . 4 5 3 5 5}$ \\
\hline \hline
\end{tabular}

crystal, the experimental data of $\chi_{z z z}^{(2)}$ are $72\left(10^{-9} \mathrm{esu}\right), \chi_{z x x}^{(2)}$ are $12\left(10^{-9} \mathrm{esu}\right)$, and $\chi_{x x x}^{(2)}$ are $6\left(10^{-9} \mathrm{esu}\right){ }^{35}$ while in $\mathrm{KNbO}_{3}, \chi_{z z z}^{(2)}$ are $53\left(10^{-9} \mathrm{esu}\right), \chi_{z x x}^{(2)}$ are $30\left(10^{-9} \mathrm{esu}\right), \chi_{z y y}^{(2)}$ are $35\left(10^{-9} \mathrm{esu}\right), \chi_{x x z}^{(2)}$ are $32\left(10^{-9} \mathrm{esu}\right)$, and $\chi_{y y z}^{(2)}$ are 33 $\left(10^{-9} \mathrm{esu}\right) .{ }^{35}$ In the mean time, for their large SHG, the linear electro-optic coefficients are also very significant. These linear electro-optic coefficients indicate that when an electric field is applied, a large change of refraction can be observed in $\mathrm{Bi}_{2} \mathrm{ZnTiO}_{6}$.

Optical calculations based on the local density approximation (LDA) or GGA might overestimate the SHG coefficients mainly because of their underestimation of the band gap. Therefore, to confirm the large $\mathrm{SHG}$ in $\mathrm{Bi}_{2} \mathrm{ZnTiO}_{6}$ mentioned above, we have further performed the $a b$ initio calculations of the $\mathrm{SHG}$ in $\mathrm{LiNbO}_{3}$ by using the same computational method described in Sec. II. The PAW potentials used are with three valence electrons for $\mathrm{Li}\left(1 s^{2} 2 s^{1}\right)$ and 13 for $\mathrm{Nb}$ $\left(4 s^{2} 4 p^{6} 4 d^{4} 5 s^{1}\right)$. In the self-consistent band structure and optical properties calculations, a dense $k$-point grid $16 \times 16$ $\times 16$ is adopted with $417 k$-points in the irreducible Brillouin zone. The calculated largest static component of $\chi^{(2)}$ is $62 \times 10^{-9} \mathrm{esu}$, which is consistent with experimental data $\left(72 \times 10^{-9} \mathrm{esu}\right)$. This lends support to our finding of the large SHG in $\mathrm{Bi}_{2} \mathrm{ZnTiO}_{6}$ reported above. Moreover, we have also performed the LDA optical calculations for $\mathrm{Bi}_{2} \mathrm{ZnTiO}_{6}$ in the different $B$-site orderings, and found that the calculated dielectric functions and SHG coefficients (not shown here) are very similar to the corresponding GGA results displayed in Figs. 2 and 9. Therefore, we may conclude that $\mathrm{Bi}_{2} \mathrm{ZnTiO}_{6}$ possesses the largest static SHG coefficients than other known ferroelectric oxides. Furthermore, such large static nonlinear optic coefficients are found in all types of $B$-site ordering, thus being almost independent of $B$-site ordering.

As indicated in Eq. (4), the larger electric polarization, the larger nonlinear optical susceptibilities. In these highly distorted perovskite systems, the electric polarization is directly related to the tetragonality $c / a$ as well as the displacement of ferroelectric atoms away from their centrosymmetric positions. To have a picture of the relationship between the polar structure and large nonlinear optical responses, we list the exact value of $c / a$ ratio, bonding lengths, and the offcenter displacement of $\mathrm{Bi}, \mathrm{Zn}$, and Ti atoms in Table III. It is found that the largest $c / a$ is found in $C$-type system, which reaches 1.331, while the energetically favorable $G$-type has a $c / a$ ratio of 1.304, and $A$-type system of 1.315. Such kind of large tetragonality is rare $\left(\mathrm{PbTiO}_{3}\right.$ has $c / a$ ratio of 1.06), and only two antiferromagnetic $\mathrm{BiCoO}_{3}$ and $\mathrm{PbVO}_{3}$ exhibit similar large polar distortions which are 1.27 and 1.23 , respectively. The large $c / a$ ratio in perovskite is produced by a distortion of the $B$-site coordination geometry from tetragonally elongated octahedral to square based pyramidal, coupled with an $A$-site bismuth environment also considerable more distorted than that of $\mathrm{PbTiO}_{3}$, reflecting a more pronounced influence of the $6 s^{2}$ lone pair. As for the bonding lengths, the longest $\mathrm{Ti}-\mathrm{O}$ bond $(3.2090 \AA)$ is found in the $C$-type system. The longest $\mathrm{Zn}-\mathrm{O}$ bond $(3.5265 \AA)$ is found in the $A$-type system. For $\mathrm{Bi}$ ions, the largest bond length appears in the $G$-type system. Consequently, significant offcenter displacements of $\mathrm{Zn}, \mathrm{Ti}$, and $\mathrm{Bi}$ atoms are found. In particular, a large off-center displacement is found in $\mathrm{Zn}$ atoms for the first time. This finding is much larger than previous $a b$ initio results performed in $\left[\mathrm{Bi}_{2} \mathrm{ZnTiO}_{6}\right]_{x}\left[\mathrm{PbTiO}_{3}\right]_{1-x}$ alloys, which shows an increase in $c / a$ and off-center displacement as the increase in $x .^{27} \mathrm{In}$ $\mathrm{PbTiO}_{3}$, the off-center of Ti atom is $0.32 \AA$ and that of $\mathrm{Pb}$ is $0.48 \AA$, much smaller than $\mathrm{Bi}_{2} \mathrm{ZnTiO}_{6}$ as revealed here. In the mean time, it is noted that in both $G$-type and $A$-type systems, the off-center displacement of $\mathrm{Zn}$ atom is larger than that of atom $\mathrm{Ti}$, indicating an enhanced ferroelectric driving force from the $\mathrm{Zn}$ site. These calculated structure data have supplemented current experimental data, ${ }^{19}$ which could not give ample information on the $\mathrm{Zn}$, Ti, as well as Bi environments.

It is noted that there is no direct evidence of ferroelectric behavior in $\mathrm{Bi}_{2} \mathrm{ZnTiO}_{6}$ from current experiment. ${ }^{19}$ This is similar with $\mathrm{PbVO}_{3}$ and $\mathrm{BiCoO}_{3}$, which also exhibit very large polar distortion. In such materials, exceptionally large electric field required for switching makes it very difficult to obtain a $P-E$ hysteresis loop. This point is verified by our total energy calculations of $\mathrm{Bi}_{2} \mathrm{ZnTiO}_{6}$ in centrosymmetric 


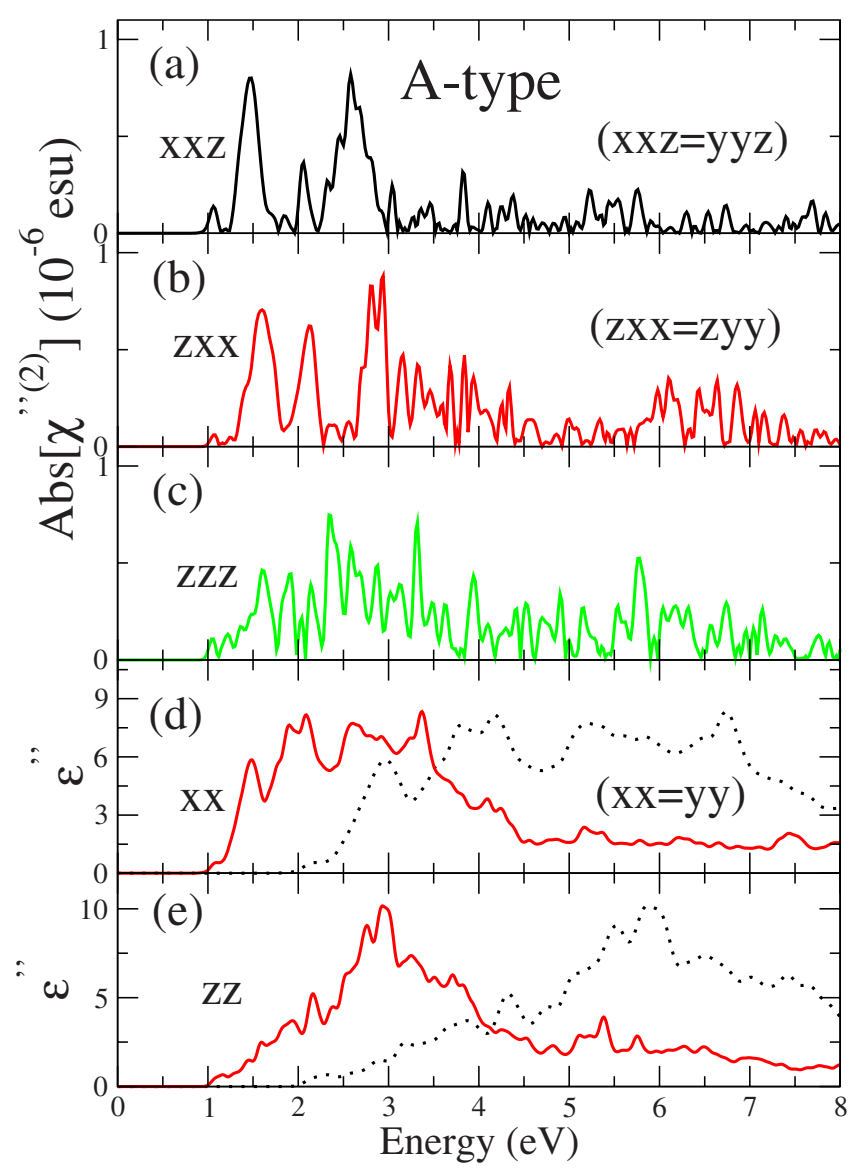

FIG. 3. (Color online) Absolute value of the imaginary part of $\chi^{(2)}$ as well as imaginary part of dielectric functions $\varepsilon(\omega)$ and $\varepsilon(\omega / 2)$ of $A$-type system.

cubic phase, which shows almost $5 \mathrm{eV}$ higher per formula unit than their polar structures. Nevertheless the nonlinear optical properties as revealed above provide a direction of its potential application.

It is generally believed that the driving force of such unusual polar structure in the $A$-site driven ferroelectrics, such as $\mathrm{BiFeO}_{3}, \mathrm{BiAlO}_{3}, \mathrm{BiInO}_{3}$, and present $\mathrm{Bi}_{2} \mathrm{ZnTiO}_{6}$ is due to the $\mathrm{Bi} 6 s^{2}$ lone pair, which can be regarded as $6 p^{0}$-ness. Therefore, the electron localized function ${ }^{36}$ is displayed for visualizing localized Bi $6 s^{2}$ lone pair (see Fig. 6). Similar with other bismuth based polar oxides, the lobe shaped electrons lead to anisotropic bondings between surrounding oxygen atoms. In fact, the $\mathrm{Bi} 6 p^{0}$-ness is also strengthened by the presence of $B$-site $\mathrm{Ti}$ and $\mathrm{Zn}$ off-center displacements, which are due to the hybridization between $\mathrm{Ti}$ and $\mathrm{O}\left(3 d^{0}\right.$-ness), and the strong hybridization of $\mathrm{Zn}$ ( $4 s$ and $4 p$ ) and $\mathrm{O}$, respectively. This point will be evaluated from the local DOS displayed below. Therefore, from the above analysis, the highly distorted structure in $\mathrm{Bi}_{2} \mathrm{ZnTiO}_{6}$ arises from both $A$-site and $B$-site.

It is clear from Eqs. (9) and (10) that the SHG coefficients can have pronounced features due to both single- and double-frequency resonant terms. To further understand the microscopic origin of such large SHG, here we plot the absolute value of imaginary part of $\chi^{(2)}$ in comparison with the imaginary part of $\varepsilon^{\prime \prime}(\omega)$ and $\varepsilon^{\prime \prime}(\omega / 2)$. The $P 4 m m$ symmetry of $\mathrm{Bi}_{2} \mathrm{ZnTiO}_{6}$ allows $\varepsilon_{x x}=\varepsilon_{y y}$. For the $A$-type system (Fig. 3),

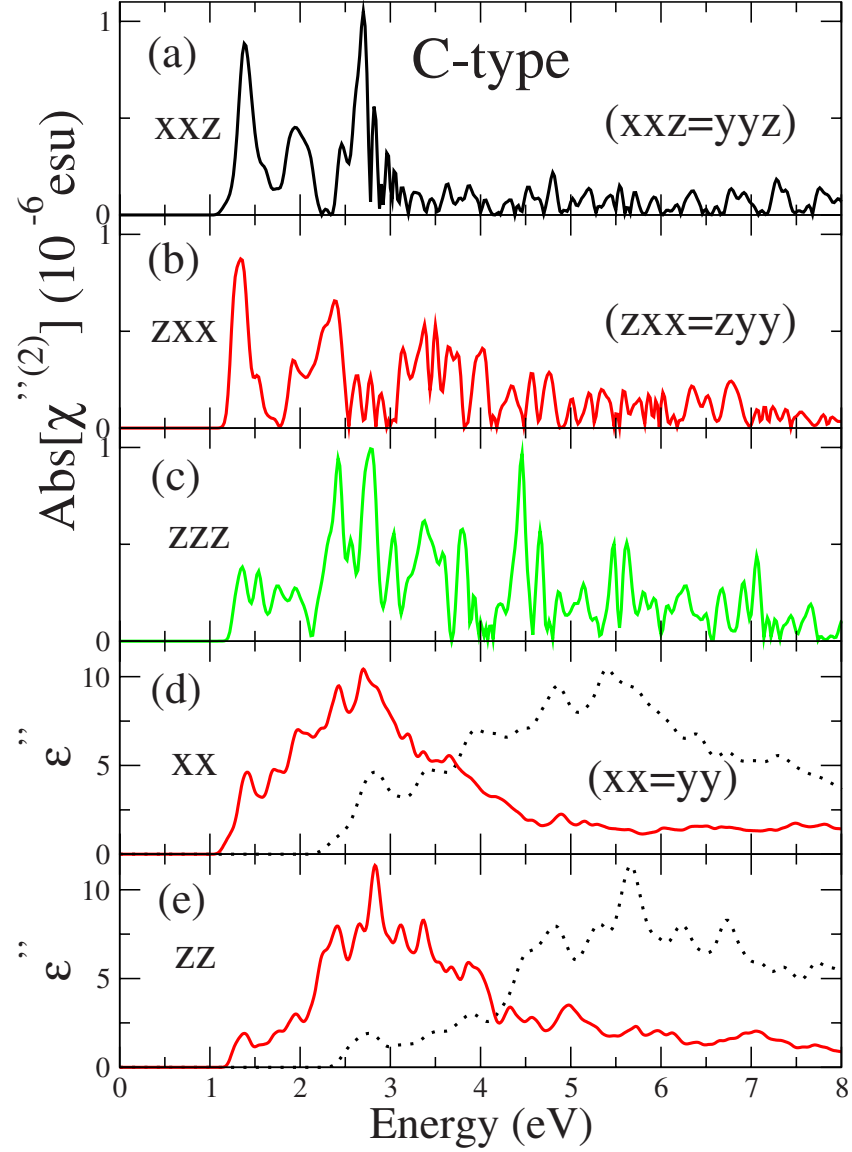

FIG. 4. (Color online) Absolute value of the imaginary part of $\chi^{(2)}$ as well as imaginary part of dielectric functions $\varepsilon(\omega)$ and $\varepsilon(\omega / 2)$ of $C$-type system.

the first peak observed around $1.5 \mathrm{eV}$ in $\chi_{x x z}^{(2)}$ and $\chi_{z x x}^{(2)}$ are almost identical to the first peak in $\varepsilon^{\prime \prime}(\omega / 2)_{x x}$. The second peak around $2.1 \mathrm{eV}$ in $\chi_{x x z}^{(2)}$ and $\chi_{z x x}^{(2)}$ is also similar with the second peak in $\varepsilon^{\prime \prime}(\omega / 2)_{x x}$. The third peak in $\chi_{x x z}^{(2)}$ around $2.6 \mathrm{eV}$ is consistent with a broad peak in this range of $\varepsilon^{\prime \prime}(\omega / 2)_{x x}$. All these can be regarded as two-photon resonances, while the third peak in $\chi_{z x x}^{(2)}$ around $2.9 \mathrm{eV}$ is consistent with the first peak in $\varepsilon^{\prime \prime}(\omega)_{x x}$, indicating single-photon resonances. When the energy is higher than $3 \mathrm{eV}$, SHG decreased and exhibit obscure peaks. For the $C$-type system (Fig. 4), almost the same trend in A-type is found, i.e., for the low energy photon (less than $3 \mathrm{eV}), \chi^{(2)}$ is directly related to $\varepsilon^{\prime \prime}(\omega / 2)$. For higher energy photon, peaks in $\chi^{(2)}$ are almost the same as that in $\varepsilon^{\prime \prime}(\omega)$. Again, for the $G$-type system (Fig. 5), when energy is less than $3 \mathrm{eV}$, SHG peaks in $\chi_{x x z}^{(2)}$ and $\chi_{z x x}^{(2)}$ are directly related with the four peaks in $\varepsilon^{\prime \prime}(\omega / 2)_{x x}$, indicating two-photon resonances. The same is found in $\chi_{z z z}^{(2)}$ with three dominating peaks in this energy range. When the energy is larger than $3 \mathrm{eV}$, SHG is related with $\varepsilon^{\prime \prime}(\omega / 2)$, which indicates single-photon resonance. Therefore, the successful connection of the prominent feature of SHG in $\mathrm{Bi}_{2} \mathrm{ZnTiO}_{6}$ with that of the linear optical dielectric function in terms of single-photon and double-photon resonances indicates the source of large $\mathrm{SHG}$ in $\mathrm{Bi}_{2} \mathrm{ZnTiO}_{6}$.

The optical properties can be analyzed further from DOS. In Fig. 7, we have shown the total and partial DOSs. The basic shape is the same for three types of $B$-site order- 


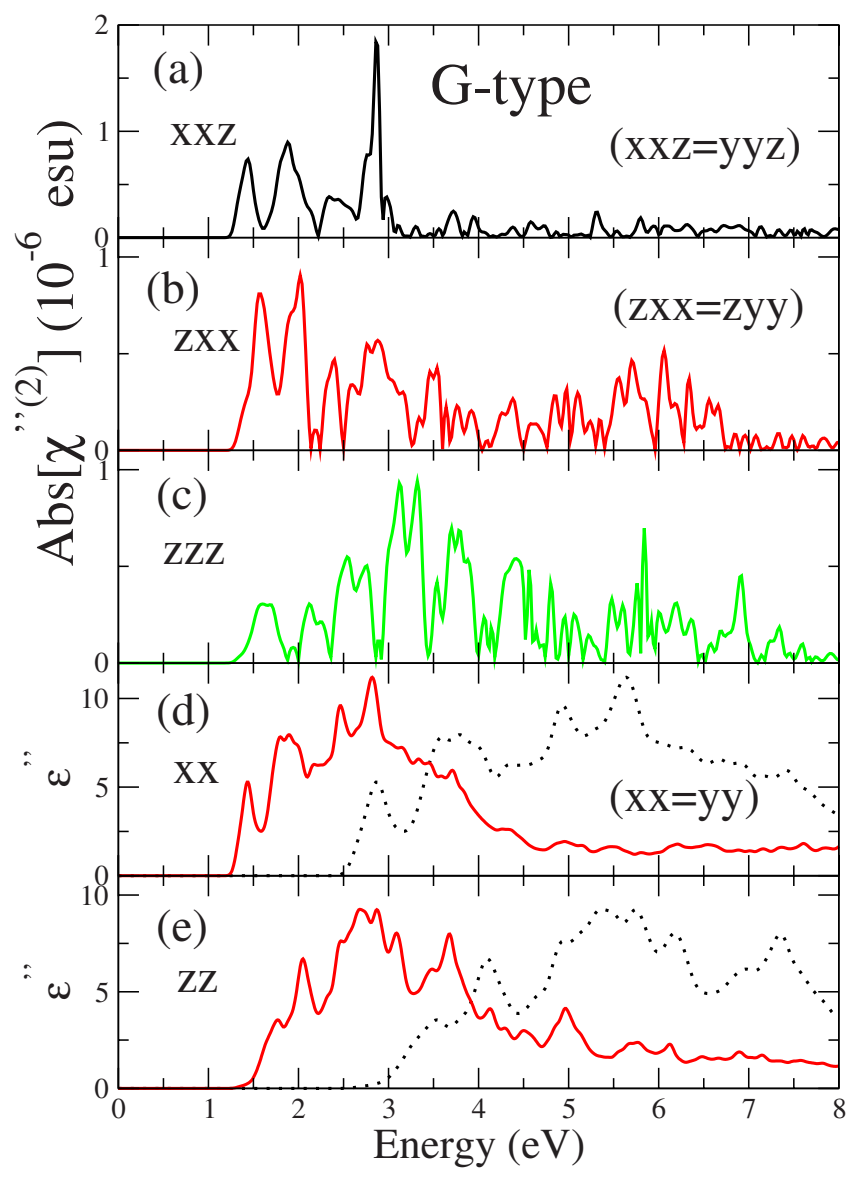

FIG. 5. (Color online) Absolute value of the imaginary part of $\chi^{(2)}$ as well as imaginary part of dielectric functions $\varepsilon(\omega)$ and $\varepsilon(\omega / 2)$ of $G$-type system.

ing, but with different bandgaps. From $A$-type to $G$-type, the band gap increases from around 1 to $1.5 \mathrm{eV}$. In all three types of ordering, the lowest bands at $-10 \mathrm{eV}$ are mainly from $\mathrm{Bi} 6 s$ states. Above these bands are the bands of the hybridized $\mathrm{O} p$, Ti $p$, and $\mathrm{Zn} 4 p, 4 s$ states. The conduction band is of Ti $d$ states as well as the band of the Bi $6 p$ states at higher energy. The hybridization between $\mathrm{Bi}$ and $\mathrm{O}$, Ti and

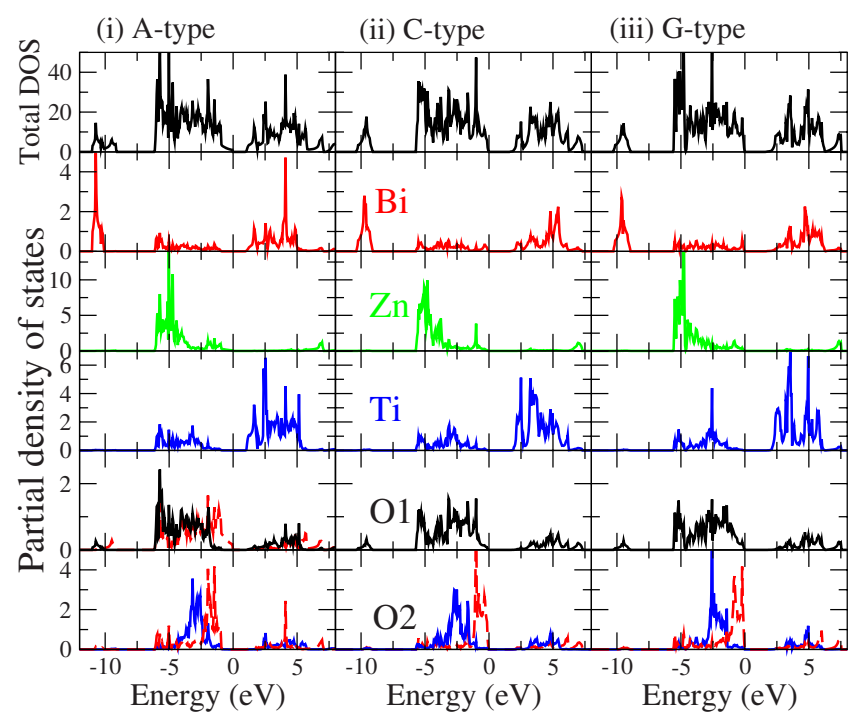

FIG. 7. (Color online) Total and partial density of states of three types of $\mathrm{Bi}_{2} \mathrm{ZnTiO}_{6}$. (i) $A$-type. (ii) $C$-type. (iii) $G$-type.

$\mathrm{O}\left(3 d^{0}\right.$-ness), and $\mathrm{Zn}(4 s$ and $4 p)$ and $\mathrm{O}$ are the driving forces of the polar structures. The detailed band structure is plotted in Fig. 8. It is noted that $A$-type has a direct band gap on $\Gamma$ point, while the other two types have indirect band gap.

Finally, we display in Fig. 9 the linear optical responses which can be easily accessible in experiments and will verify our theoretical prediction in turn. In Fig. 9, from left to right, we present the results of $A$-type, $C$-type, and $G$-type, respectively. Both $x x$ and $z z$ components are displayed. The difference between the three types of $B$-site ordering is not as obvious as SHG. The imaginary part of dielectric function in Fig. 9(b) shows the interband transition peaks. The detailed refraction function is displayed in Fig. 9(c) and the static refraction indices for $A$-type are 2.56 and 2.39 for $x x$ and $z z$ components, respectively. For $C$-type, they are 2.57 and 2.40 , respectively. And for $G$-type, they are 2.57 and 2.40 , respectively. As expected from the imaginary part of $\varepsilon$, the absorption spectrum in Fig. 9(d) shows the two broad peaks

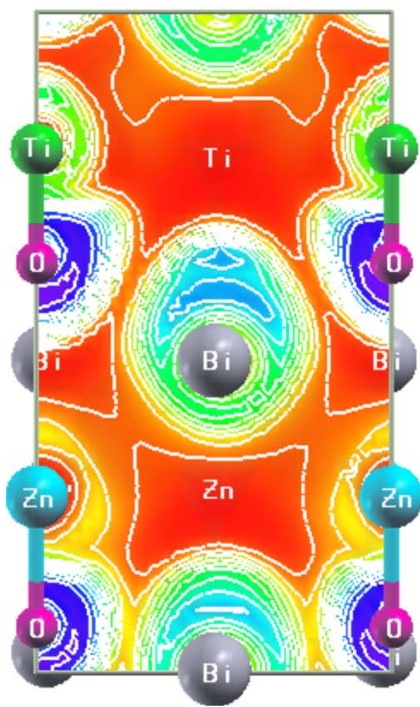

(a)

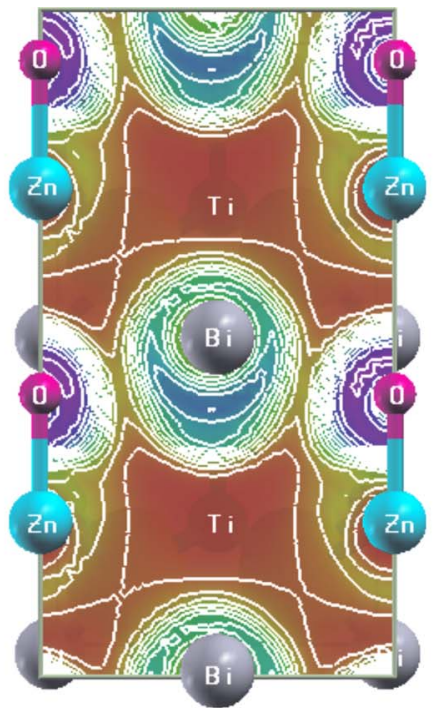

(b)

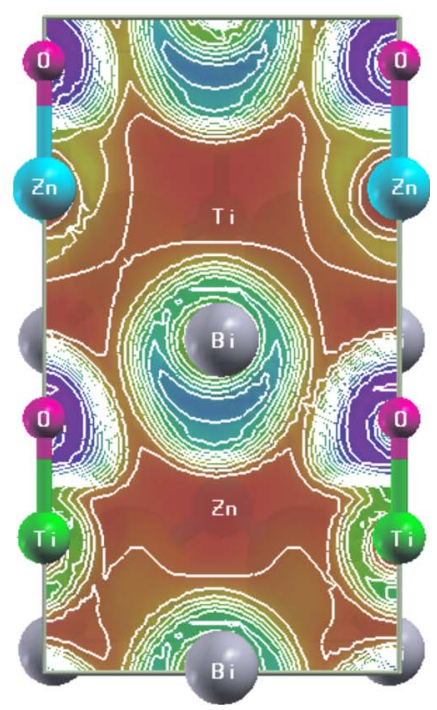

(c)

FIG. 6. (Color online) Illustration of electron localization function. (a) A-type. (b) $C$-type. (c) $G$-type. 
(a) A-type

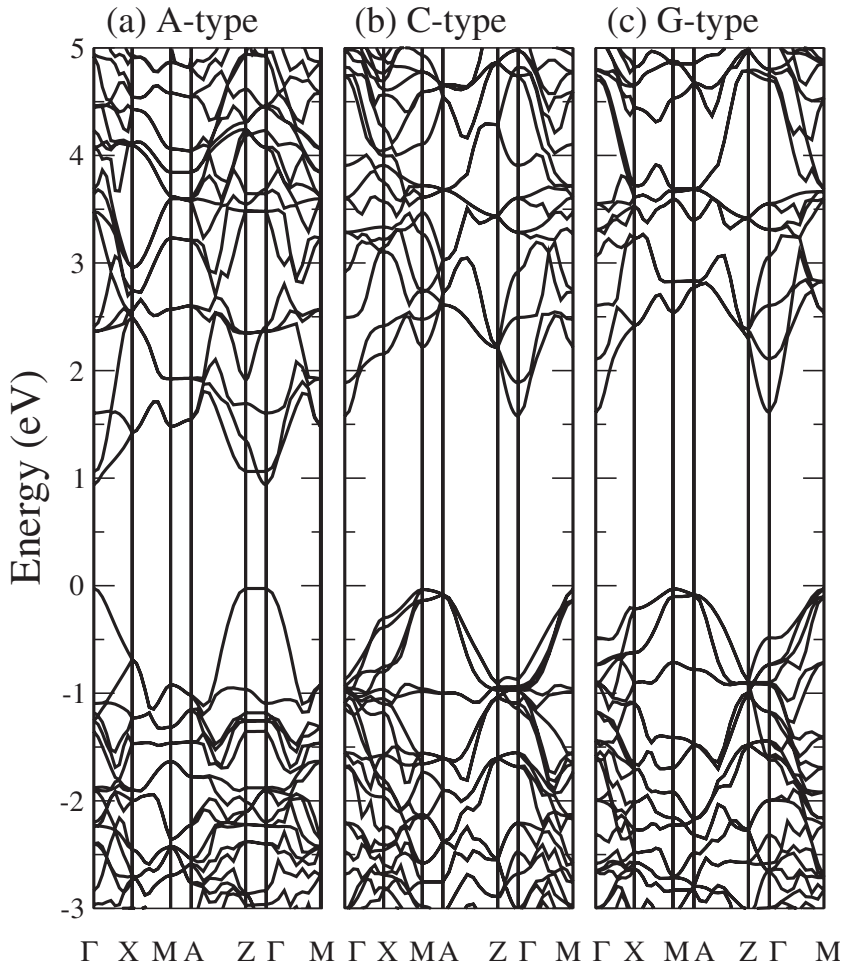

\section{SUMMARY}

In summary, we have made a systematic $a b$ initio investigation of crystal structure, electronic structure, linear optical, and SHG in $\mathrm{Bi}_{2} \mathrm{ZnTiO}_{6}$. By comparing three possible $B$-site ordering configurations, we have found the following: (i) $G$-type $B$-site ordering is energetically favorable, (ii) all the three types of ordering show large SHG coefficients, and (iii) the largest static $\mathrm{SHG}$ appears in $C$-type $\mathrm{Bi}_{2} \mathrm{ZnTiO}_{6}$ with the value of $123 \times 10^{-9}$ esu, almost two times larger than $72 \times 10^{-9}$ esu in $\mathrm{LiNbO}_{3}$. The significant SHG coefficients are consistent with the calculated unusual polar structures, i.e., large $c / a$ ratio and off-center displacements, and are further related to the linear part of optical dielectric function in terms of single-photon and double-photon resonances. These results have demonstrated the potential application of $\mathrm{Bi}_{2} \mathrm{ZnTiO}_{6}$ in future optoelectronics. We hope our calculations will stimulate further experiments on the SHG measurements in this unusual polar double perovskite oxide.
FIG. 8. Band structure of three types of $\mathrm{Bi}_{2} \mathrm{ZnTiO}_{6}$. (a) $A$-type. (b) $C$-type (c) $G$-type.

(around 7.5 and $17.5 \mathrm{eV}$ ) and a sharp one $(27.5 \mathrm{eV})$. The energy-loss function describes the energy loss of a fast electron traversing in a material and is large at the plasmon energy. The energy-loss function shown in Fig. 9(e) displays two broad peaks and a sharp one, located around 10, 20, and $28 \mathrm{eV}$, respectively, which are consistent with the roots of $\varepsilon^{\prime}(\omega)$. These linear optical results will provide useful information for experimentalists.

\section{ACKNOWLEDGMENTS}

The authors gratefully acknowledge support from the National Science Council of Taiwan, National Center for Theoretical Sciences of Taiwan, TSMC under TSMC JDP \#NTU-8606, and National Natural Science Foundation of China under Grant Nos. 10504023 and 10774107.
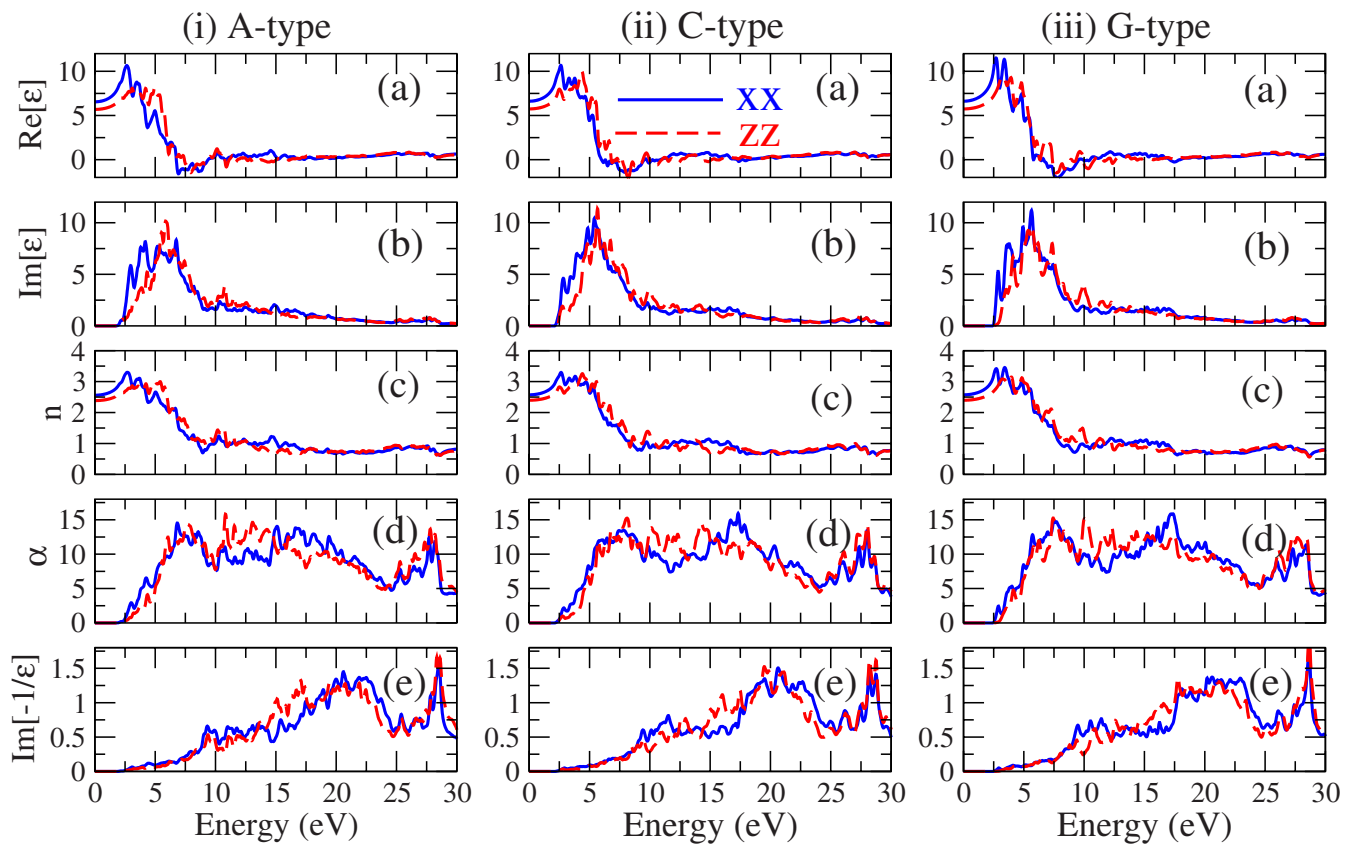

FIG. 9. (Color online) Linear optical properties. From left to right are $A$-type, $C$-type, and $G$-type, respectively. (a) Real part of $\varepsilon$. (b) Imaginary part of $\varepsilon$. (c) Refraction spectrum. (d) Absorption spectrum. (e) EELS function. Blue solid line is for $\varepsilon_{x x}$ and red dashed line is $\varepsilon_{z z}$. For the $P 4 m m$ symmetry, $\varepsilon_{x x}=\varepsilon_{y y}$. 
${ }^{1}$ K.-I. Kobayashi, T. Kimura, H. Sawada, K. Terakura, and Y. Tokura, Nature (London) 395, 677 (1987).

${ }^{2}$ N. S. Rogado, J. Li, A. W. Sleight, and M. A. Subramanian, Adv. Mater. (Weinheim, Ger.) 17, 2225 (2005).

${ }^{3}$ Y. K. Wang and G. Y. Guo, Phys. Rev. B 73, 064424 (2006).

${ }^{4}$ R. Nechache, C. Harnagea, A. Pignolet, F. Normandin, T. Veres, L.-P. Carignan, and D. Menard, Appl. Phys. Lett. 89, 102902 (2006).

${ }^{5}$ M. R. Suchomel, C. I. Thomas, M. Allix, M. J. Rosseinsky, A. M. Fogg, and M. F. Thomas, Appl. Phys. Lett. 90, 112909 (2007).

${ }^{6}$ D. H. Kim, H. N. Lee, M. D. Biegalski, and H. M. Christen, Appl. Phys. Lett. 91, 042906 (2007).

${ }^{7}$ A. A. Belik, S. Iikubo, T. Yokosawa, K. Kodama, N. Igawa, S. Shamoto, M. Azuma, M. Takano, K. Kimoto, Y. Matsui, and E. TakayamaMuromachi, J. Am. Chem. Soc. 129, 971 (2007).

${ }^{8}$ M. Sakai, A. Masuno, D. Kan, M. Hashisaka, K. Takata, M. Azuma, M. Takano, and Y. Shimakawa, Appl. Phys. Lett. 90, 072903 (2007).

${ }^{9}$ N. A. Hill, J. Phys. Chem. 104, 6694 (2000).

${ }^{10}$ M. Dawber, K. M. Rabe, and J. F. Scott, Rev. Mod. Phys. 77, 1083 (2005).

${ }^{11}$ R. E. Cohen, Nature (London) 358, 136 (1992).

${ }^{12}$ D. J. Singh, M. Ghita, M. Fornari, and S. V. Halilov, Ferroelectrics 338, 73 (2006).

${ }^{13}$ A. A. Belik, T. Wuernisha, T. Kamiyama, K. Mori, M. Maie, T. Nagai, Y. Matsui, and E. Takayama-Muromachi, Chem. Mater. 18, 133 (2006).

${ }^{14}$ A. A. Belik, S. Yu. Stefanovich, B. I. Lazoryak, and E. TakayamaMuromachi, Chem. Mater. 18, 1964 (2006).

${ }^{15}$ J. Zylberberg, A. A. Belik, E. Takayama-Muromachi, and Z.-G. Ye, Chem. Mater. 19, 6385 (2007).

${ }^{16}$ A. A. Belik, S. Iikubo, K. Kodama, N. Igawa, S. Shamoto, M. Maie, T. Nagai, Y. Matsui, S. Yu. Stefanovich, B. I. Lazoryak, and E. TakayamaMuromachi, J. Am. Chem. Soc. 128, 706 (2006).

${ }^{17}$ A. A. Belik, T. Yokosawa, K. Kimoto, Y. Matsui, and E. TakayamaMuromachi, Chem. Mater. 19, 1679 (2007).

${ }^{18}$ D. D. Khalyavin, A. N. Salak, N. P. Vyshatko, A. B. Lopes, N. M. Ole- khnovich, A. V. Pushkarev, I. I. Maroz and Y. V. Radyush, Chem. Mater. 18, 5104 (2007).

${ }^{19}$ M. R. Suchomel, A. M. Fogg, M. Allix, H. J. Niu, J. B. Claridge, and M. J. Rosseinsky, Chem. Mater. 18, 4987 (2006); Chem. Mater. 18, 5810 (2006).

${ }^{20}$ M. R. Suchomel and P. T. Davis, Appl. Phys. Lett. 86, 262905 (2005).

${ }^{21}$ R. D. King-Smith and D. Vanderbilt, Phys. Rev. B 47, 1651 (1993).

${ }^{22}$ R. Resta, Rev. Mod. Phys. 66, 899 (1994).

${ }^{23}$ P. Baettig and N. A. Spaldin, Appl. Phys. Lett. 86, 012505 (2005); P. Baettig, C. Ederer, and N. A. Spaldin, Phys. Rev. B 72, 214105 (2005).

${ }^{24}$ A. Ciucivara, B. Sahu, and L. Kleinman, Phys. Rev. B 76, 064412 (2007).

${ }^{25}$ J. B. Neaton, C. Ederer, U. V. Waghmare, N. A. Spaldin, and K. M. Rabe, Phys. Rev. B 71, 014113 (2005); C. Ederer and N. A. Spaldin, ibid. 71, 060401(R) (2005)

${ }^{26}$ P. Baettig, C. F. Schelle, R. LeSar, U. V. Waghmare, and N. A. Spaldin, Chem. Mater. 17, 1376 (2005).

${ }^{27}$ J. Iniguez, D. Vanderbilt, and L. Bellaiche, Phys. Rev. B 67, 224107 (2007); I. Grinberg, M. R. Suchomel, W. Dmowski, S. E. Mason, H. Wu, P. K. Davies, and A. M. Rappe, Phys. Rev. Lett. 98, 107601 (2007).

${ }^{28}$ P. E. Blochl, Phys. Rev. B 50, 17953 (1994).

${ }^{29}$ G. Kresse and J. Hafner, Phys. Rev. B 47, 558 (1993); 49, 14251 (1994); G. Kresse and J. Furthmuller, Comput. Mater. Sci. 6, 15 (1996); G. Kresse and D. Joubert, Phys. Rev. B 59, 1758 (1999).

${ }^{30}$ P. E. Blochl, O. Jepsen, and O. K. Andersen, Phys. Rev. B 49, 16223 (1994).

${ }^{31}$ G. Y. Guo, K. C. Chu, D. S. Wang, and C. G. Duan, Phys. Rev. B 69, 205416 (2004).

${ }^{32}$ G. Y. Guo and J. C. Lin, Phys. Rev. B 71, 165402 (2005).

${ }^{33}$ G. Y. Guo and J. C. Lin, Phys. Rev. B 72, 075416 (2005); 77, 049901(E) (2008).

${ }^{34}$ R. W. Boyd, Nonlinear Optics (Elsevier, Amsterdam, 2003).

${ }^{35}$ W.-L. Zhong, Ferroelectrics (Chinese Academy, Beijing, 2000).

${ }^{36}$ B. Silvi and A. Savin, Nature (London) 371, 683 (1994). 